\title{
Toy models of developed turbulence
}

\author{
M.Hnatich, M.Jurcisin, M.Repasan \\ Institute of Experimental Physics, \\ Dept. Theor.Phys., \\ Kosice, Watsonova 47, Slovakia
}

Received November 11, 2004

\begin{abstract}
We have investigated the advection of a passive scalar quantity by incompressible helical turbulent flow within the framework of extended Kraichnan model. Turbulent fluctuations of velocity field are assumed to have the Gaussian statistics with zero mean and defined noise with finite timecorrelation. Actual calculations have been done up to two-loop approximation within the framework of field-theoretic renormalization group approach. It turned out that space parity violation (helicity) of turbulent environment does not affect anomalous scaling which is a peculiar attribute of the corresponding model without helicity. However, stability of asymptotic regimes, where anomalous scaling takes place, strongly depends on the amount of helicity. Moreover, helicity gives rise to the turbulent diffusivity, which has been calculated in one-loop approximation.
\end{abstract}

Key words: helicity, diffusivity, anomalous scaling, renormalization group

PACS: 47.10.+g, 47.27.-i, 05.10.Cc

\section{Introduction}

One of the main problems in the modern theory of fully developed turbulence is to verify the basic principles of the celebrated Kolmogorov-Obukhov (KO) phenomenological theory [1-3] within the framework of a microscopic model and to investigate possible deviations from this theory. Turbulent flows that occur in various liquids or gases at very high Reynolds numbers reveal a number of general aspects (cascades of energy or other conserved quantities, scaling behavior with apparently universal "anomalous exponents," intermittency, statistical conservation laws and so on), which support the hopes that those phenomena can be explained within a self-contained and internally consistent theory. Recent developments in this area are presented and summarized in [4].

The most remarkable features of developed turbulence are encoded in the single term of intermittency. Roughly speaking, intermittency means that statistical properties (for example, correlation or structure functions of the turbulent velocity field) are dominated by rare spatiotemporal configurations, in which the regions 
with strong turbulent activity have exotic (fractal) geometry and are embedded into the vast regions with regular (laminar) flow. In the turbulence, such phenomenon is believed to be related to strong fluctuations of the energy flux. Therefore, it leads to deviations from the predictions of the aforementioned KO theory. Such deviations, referred to as "anomalous" or "non-dimensional" scaling, manifest themselves in singular (arguably power-like) dependence of correlation or structure functions on the distances and the integral (external) turbulence scale $L$. The corresponding exponents are certain nontrivial and nonlinear functions of the order of the correlation function, i.e., the phenomenon referred to as "multiscaling."

The term "anomalous scaling" reminds of the critical scaling in models of equilibrium phase transitions. In those, the field theoretic methods were successfully employed to establish the existence of self-similar (scaling) regimes and to construct regular perturbative calculational schemes (the famous $\epsilon$ expansion and its relatives) for the corresponding exponents, scaling functions, ratios of amplitudes etc; see e.g. $[5,6]$. Here and below, by "field theoretic methods" we mean diagrammatic and functional techniques, renormalization theory and renormalization group, composite operators, operator-product expansion and so on.

Although the theoretical description of the fluid turbulence based on the "first principles" i.e. on the stochastic Navier-Stokes (NS) equations [1] remains essentially an open problem, considerable progress has been achieved in understanding simplified model systems that share some important properties with the real problem: shell models [7], stochastic Burgers equation [8] and passive advection by random "synthetic" velocity fields [9].

Probably the most important progress in the subject, achieved in the last decade of the twentieth century, was related to a simplified model of the fully developed turbulence, the so-called rapid-change model. The model, which dates back to classical studies of Batchelor, Obukhov, Kraichnan and Kazantsev, describes a scalar or vector quantity (e.g. temperature, concentration of admixture particles or a weak magnetic field), passively advected by a Gaussian velocity field, decorrelated in time and self-similar in space (the latter property mimics some features of a real turbulent velocity ensemble). There, for the first time the existence of anomalous scaling was established based on the microscopic model [10], and the corresponding anomalous exponents were derived within controlled approximations [11,12] and regular perturbation schemes based on the field theoretic renormalization group (RG) and operator product expansion (OPE) [13]. Detailed review of the recent theoretical research on the passive scalar problem and more references can be found in [9].

The feature specific to the theory of turbulence and simplified models associated with it is the existence in the corresponding field theoretical models of the composite operators with negative scaling (critical) dimensions. Such operators, termed "dangerous" in [13-17], give rise to anomalous scaling, i.e., the singular dependence on the infrared (IR) scale $L$ with certain nonlinear anomalous exponents.

Important advantages of the RG approach are its universality and calculational efficiency: a regular systematic perturbation expansion for the anomalous exponents was constructed, similar to the well-known $\epsilon$-expansion in the theory of phase transi- 
tions, and the exponents were calculated in the first order of expansion for passively advected vector fields [18,19] and in the second [13] and third [15] orders of that expansion for scalar fields. Furthermore, the RG approach is not related only to the rapid-change model and can also be applied to the case of finite correlation time or non-Gaussian advecting field [17].

The solution proceeds in two main stages. In the first stage, the multiplicative renormalizability of the corresponding field theoretic model is demonstrated and the differential RG equations for its correlation functions are obtained. The asymptotic behavior of the latter on their UV argument $(r / l)(l$ is internal length) for $r \gg \ell$ and any fixed $(r / L)$ ( $L$ is an outer length) is given by IR stable fixed points of those equations. It involves some "scaling functions" of the IR argument $(r / L)$, whose form is not determined by the RG equations. In the second stage, their behavior at $r \ll L$ is found from the OPE within the framework of the general solution of the RG equations. The crucial role is played there by the critical dimensions of various composite operators, which give rise to an infinite family of independent scaling exponents (and hence to multiscaling). Of course, these both stages (and thus the phenomenon of multiscaling) have long been known in the RG theory of critical behavior. The distinguishing feature specific to models of turbulence is the existence of composite operators with aforementioned negative critical dimensions. Their contributions to the OPE diverge at $(r / L) \rightarrow 0$. In the models of critical phenomena, nontrivial composite operators always have strictly positive dimensions, so that they only determine corrections (vanishing for $(r / L) \rightarrow 0$ ) to the leading terms (finite for $(r / L) \rightarrow 0$ ) in the scaling functions.

Regular perturbation schemes and accurate numerical simulations allow one to discuss, taking the example of the rapid-change model and its relatives, the issues that are interesting within the general context of fully developed turbulence: universality and saturation of anomalous exponents, effects of compressibility, anisotropy and pressure, persistence of the large-scale anisotropy and hierarchy of anisotropic contributions and so on. Moreover, it is interesting and important to study the helicity (violation of space parity) effects because many turbulence phenomena are directly effected by them (like large air vortices in atmosphere). For example, in the framework of turbulent magnetohydrodynamics it leads to a nontrivial fact of the very existence of the so-called "turbulent dynamo", i.e., the generation of a large-scale magnetic field by the energy of the turbulent motion [20-24]. This is an important effect in astrophysics.

Helicity, as we shall see below, does not affect the known results in one-loop approximation. Therefore, it is necessary to explore the second order (two-loop) approximation. It is also important to say that in the framework of classical Kraichnan model, i.e., the model of passive advection by Gaussian velocity field with $\delta$-like correlations in time, it is impossible to study the effect of helicity since all potentially "helical" diagrams are identically equal to zero at all orders in the perturbation theory. In this sense, the investigation of helicity in the present model can be considered as the first step to analyze helicity in the problems with genuine turbulence. 


\section{Field theoretic description of the model}

The advection of a passive scalar field $\theta(x) \equiv \theta(t, \mathbf{x})$ in helical turbulent environment is described by the stochastic equation

$$
\partial_{t} \theta+v_{i} \partial_{i} \theta=\nu_{0} \Delta \theta+f
$$

where $\partial_{t} \equiv \partial / \partial t, \partial_{i} \equiv \partial / \partial x_{i}, \nu_{0}$ is the molecular diffusivity coefficient (hereafter all parameters with a subscript 0 denote bare parameters of unrenormalized theory; see below), $\triangle \equiv \partial^{2}$ is the Laplace operator, $v_{i} \equiv v_{i}(x)$ is the $i$-th component of the divergence-free (owing to the incompressibility) velocity field $\mathbf{v}(x)$, and $f \equiv f(x)$ is an artificial Gaussian random noise with zero mean and correlation function

$$
\left\langle f(x) f\left(x^{\prime}\right)\right\rangle=\delta\left(t-t^{\prime}\right) C(\mathbf{r} / L), \quad \mathbf{r}=\mathbf{x}-\mathbf{x}^{\prime},
$$

where $L$ denotes an integral (outer) scale. It maintains the steady-state of the system but the detailed form of the function $C(\mathbf{r} / L)$ is unessential in our consideration. In spite of the fact that in real problems the velocity field $\mathbf{v}(x)$ satisfies Navier-Stokes equation, in what follows, we suppose that statistics of velocity field is given in the form of Gaussian distribution with zero mean and correlator

$$
\left\langle v_{i}(x) v_{j}\left(x^{\prime}\right)\right\rangle=\int \frac{\mathrm{d} \omega \mathrm{d}^{d} k}{(2 \pi)^{d+1}} P_{i j}^{\rho}(\mathbf{k}) D_{v}(\omega, k) \exp \left[-\mathrm{i}\left(t-t^{\prime}\right)+\mathrm{ik}\left(\mathbf{x}-\mathbf{x}^{\prime}\right)\right],
$$

with

$$
D_{v}(\omega, k)=\frac{D_{0} k^{4-d-2 \varepsilon-\eta}}{\left(\mathrm{i} \omega+u_{0} \nu_{0} k^{2-\eta}\right)\left(-\mathrm{i} \omega+u_{0} \nu_{0} k^{2-\eta}\right)},
$$

where $D_{0}=g_{0} \nu_{0}^{3}$ is a positive amplitude factor, $g_{0}$ plays the role of the coupling constant of the model, an analog of the coupling constant $\lambda_{0}$ in the $\lambda_{0} \varphi^{4}$ model of critical behavior $[5,6]$. In addition, $g_{0}$ is a formal small parameter of the ordinary perturbation theory. The positive exponents $\varepsilon$ and $\eta(\varepsilon=O(\eta))$ are small RG expansion parameters, the analogs of the parameter $\varepsilon=4-d$ in the $\lambda_{0} \varphi^{4}$ theory. Thus we have a kind of double expansion model in the $\varepsilon-\eta$ plane around the origin $\varepsilon=\eta=0$. The correlator (4) is directly related to the energy spectrum via the frequency integral [17]

$$
E(k) \simeq k^{d-1} \int \mathrm{d} \omega D^{v}(\omega, k) \simeq \frac{g_{0} \nu_{0}^{2}}{u_{0}} k^{1-2 \varepsilon} .
$$

Therefore, the coupling constant $g_{0}$ and the exponent $\varepsilon$ describe the equal-time velocity correlator or, equivalently, energy spectrum. On the other hand, the constant $u_{0}$ and the second exponent $\eta$ are related to the frequency $\omega \simeq u_{0} \nu_{0} k^{2-\eta}$ which characterizes the mode $k$ [25]. Thus, in our notation, the value $\varepsilon=4 / 3$ corresponds to the well-known Kolmogorov "five-thirds law" for the spatial statistics of velocity field, and $\eta=4 / 3$ corresponds to the Kolmogorov frequency. For completeness, we retain $d$-dependence in expressions (3) and (4) ( $d$ is the dimensionality of the $\mathbf{x}$ space), although, of course, when one investigates the system with helicity, the dimension of 
the $\mathbf{x}$ space should be strictly equal to three. To include helicity, transverse projector $P_{i j}^{\rho}(\mathbf{k})$ is taken in the form

$$
P_{i j}^{\rho}(\mathbf{k})=P_{i j}(\mathbf{k})+H_{i j}(\mathbf{k})=\delta_{i j}-\frac{k_{i} k_{j}}{k^{2}}+\mathrm{i} \rho \epsilon_{i j l} \frac{k_{l}}{k} .
$$

Here $P_{i j}(\mathbf{k})=\delta_{i j}-k_{i} k_{j} / k^{2}$ represents non-helical part of the total transverse projector $P_{i j}^{\rho}(\mathbf{k})$. On the other hand, $H_{i j}(\mathbf{k})=\mathrm{i} \rho \epsilon_{i j l}\left(k_{l} / k\right)$ mimics the presence of helicity in the flow. Thus, formally, the transition to the helical fluid corresponds to the break of spatial parity, and, technically, this is expressed by the fact that the correlation function is specified in the form of mixture of a true tensor and a pseudotensor. The tensor $\epsilon_{i j l}$ is Levi-Civita's completely antisymmetric tensor of rank 3 and the real parameter $\rho$ characterizes the amount of helicity. Due to the requirement of positive definiteness of the correlation function the absolute value of $\rho$ should be in the interval $|\rho| \in\langle 0,1\rangle[20]$. Non-zero helical part proportional to $\rho$ physically expresses the existence of non-zero correlations $\langle\mathbf{v} \cdot \operatorname{rot} \mathbf{v}\rangle$.

The general model (3), (4) contains two important special cases: rapid-change model limit when $u_{0} \rightarrow \infty$ and $g_{0}^{\prime} \equiv g_{0} / u_{0}^{2}=$ const, $D_{v}(\omega, k) \rightarrow g_{0}^{\prime} \nu_{0} k^{-d-2 \varepsilon+\eta}$, and quenched (time-independent or frozen) velocity field limit which is defined by $u_{0} \rightarrow 0$ and $g_{0}^{\prime \prime} \equiv g_{0} / u_{0}=$ const, $D_{v}(\omega, k) \rightarrow g_{0}^{\prime \prime} \nu_{0}^{2} k^{-d+2-2 \varepsilon}$, which is similar to the well-known models of the random walks in random environment with long range correlations; see, e.g., [26,27].

The stochastic problem (1)-(4) can be treated as a field theory with an action functional

$$
S\left(\theta, \theta^{\prime}, \mathbf{v}\right)=\frac{\theta^{\prime} D_{\theta} \theta^{\prime}}{2}+\theta^{\prime}\left[-\partial_{t}+\nu_{0} \triangle-\left(v_{i} \partial_{i}\right)\right] \theta-\frac{\mathbf{v} D_{v}^{-1} \mathbf{v}}{2}
$$

where $\theta^{\prime}$ is an auxiliary scalar field, and $D_{\theta}$ and $D_{v}$ are correlators (2) and (3), respectively. In the action (7), all the required integrations over $x=(t, \mathbf{x})$ and summations over the vector indices are quite understandable.

\section{Renormalization group analysis}

The model (7) is logarithmic for $\varepsilon=\eta=0$ (the coupling constant $g_{0}$ is dimensionless) and, in this case, possible ultraviolet (UV) divergences have the form of poles in various linear combinations of $\varepsilon$ and $\eta$ in the correlation functions. Using the standard analysis of quantum field theory one finds that all divergences can be removed by the only counterterm of the form $\theta^{\prime} \triangle \theta$ [17]. Thus, the model is multiplicatively renormalizable, which is expressed explicitly in the multiplicative renormalization of the parameters $g_{0}, u_{0}$, and $\nu_{0}$ in the form

$$
\nu_{0}=\nu Z_{\nu}, \quad g_{0}=g \mu^{2 \varepsilon+\eta} Z_{g}, \quad u_{0}=u \mu^{\eta} Z_{u} .
$$

Here the dimensionless parameters $g$, $u$, and $\nu$ are the renormalized counterparts of the corresponding bare ones, $\mu$ is the renormalization mass and $Z_{i}=Z_{i}(g, u ; d, \rho ; \varepsilon)$ 
are renormalization constants which in minimal substraction (MS) scheme have the form " $1+$ only poles in $\varepsilon "$ up to two-loop approximation.

The renormalized action functional has the following form

$$
S_{R}\left(\theta, \theta^{\prime}, \mathbf{v}\right)=\frac{\theta^{\prime} D_{\theta} \theta^{\prime}}{2}+\theta^{\prime}\left[-\partial_{t}+\nu Z_{1} \triangle-(v \partial)\right] \theta-\frac{\mathbf{v} D_{v}^{-1} \mathbf{v}}{2}
$$

where the correlator $D_{v}$ is written in renormalized parameters (in wave-numberfrequency representation). By comparison of the renormalized action (9) with definitions of the renormalization constants $Z_{i}, i=g, u, \nu(8)$ one comes to the relations among them:

$$
Z_{\nu}=Z_{1}, \quad Z_{g}=Z_{\nu}^{-3}, \quad Z_{u}=Z_{\nu}^{-1}
$$

The second and third relations are caused by the absence of the renormalization of the term with $D_{v}$ in renormalized action (9).

The issue of interest is, in particular, the behavior of the equal-time structure functions

$$
S_{n}(r) \equiv\left\langle\left[\theta(t, \mathbf{x})-\theta\left(t, \mathbf{x}^{\prime}\right)\right]^{n}\right\rangle
$$

in the inertial range, specified by the inequalities $l \ll r \ll L$ ( $l$ is internal length). Here parentheses \langle\rangle mean functional average over fields $\theta, \theta^{\prime}, \mathbf{v}$ with weight $\exp \left(S_{R}\right)$. In the isotropic case, the odd functions $S_{2 n+1}$ vanish, while for $S_{2 n}$, simple dimensionality considerations give

$$
S_{2 n}(r)=\nu_{0}^{-n} r^{2 n} R_{2 n}\left(\frac{r}{l}, \frac{r}{L}, g_{0}, u_{0}, \rho\right),
$$

where $R_{2 n}$ are some functions of dimensionless variables. In principle, they can be calculated within the ordinary perturbation theory (i.e., as series in $g_{0}$ ), but this is not beneficial in studying the inertial-range behavior: the coefficients are singular in the limits $r / l \rightarrow \infty$ and/or $r / L \rightarrow 0$, which compensate the smallness of $g_{0}$, and in order to find the correct IR behavior we have to sum the entire series. The desired summation can be accomplished using the field theoretic renormalization group (RG) and operator product expansion (OPE); see $[13,15,17]$.

The RG analysis consists of two main stages. At the first stage, the multiplicative renormalizability of the model is demonstrated and the differential RG equations for its correlation (structure) functions are obtained. The asymptotic behavior of the functions like (11) for $r / l \gg 1$ and any fixed $r / L$ is given by IR stable fixed points (see below) of the RG equations and has the form

$$
S_{2 n}(r)=\nu_{0}^{-n} r^{2 n}\left(\frac{r}{l}\right)^{-\gamma_{n}} R_{2 n}\left(\frac{r}{L}, \rho\right), \quad r / l \gg 1
$$

with certain, as yet unknown, "scaling functions" $R_{2 n}(r / L, \rho)$. In the theory of critical phenomena $[5,6]$ the quantity $\Delta\left[S_{2 n}\right] \equiv-2 n+\gamma_{n}$ is termed the "critical dimension", while the exponent $\gamma_{n}$, the difference between the critical dimension $\Delta\left[S_{2 n}\right]$ and the "canonical dimension" $-2 n$, is called the "anomalous dimension." In the case at hand, the latter has an extremely simple form: $\gamma_{n}=n \varepsilon$. Whatever be the functions $R_{2 n}(r / L, \rho)$, the representation (13) implies the existence of a scaling (scale 
invariance) in the IR region $(r / l \gg 1, r / L$ fixed) with definite critical dimensions of all "IR relevant" parameters, $\Delta\left[S_{2 n}\right]=-2 n+n \varepsilon, \Delta_{r}=-1, \Delta_{L^{-1}}=1$ and fixed "irrelevant" parameters $\nu_{0}$ and $l$.

At the second stage, the small $r / L$ behavior of the functions $R_{2 n}(r / L, \rho)$ is studied within the general representation (13) using the OPE. It shows that, in the limit $r / L \rightarrow 0$, the functions $R_{2 n}(r / L, \rho)$ have the asymptotic forms

$$
R_{2 n}(r / L)=\sum_{F} C_{F}\left(\frac{r}{L}\right)\left(\frac{r}{L}\right)^{\Delta_{n}},
$$

where $C_{F}$ are coefficients regular in $r / L$. In general, the summation is implied over certain renormalized composite operators $F$ with critical dimensions $\Delta_{n}$. In the case under consideration the leading operators $F$ have the form $F_{n}=\left(\nabla_{i} \theta \nabla_{i} \theta\right)^{n}$.

We have performed the complete two-loop calculation of the critical dimensions of the composite operators $F_{n}$ for arbitrary values of $n, d, u$ and $\rho$ :

$$
\Delta[F]=\Delta_{n}^{(1)} \varepsilon+\Delta_{n}^{(2)} \varepsilon^{2},
$$

where

$$
\Delta_{n}^{(1)}=\frac{-n(n-2)(d-1)}{2(d-1)(d+2)}
$$

is the expression obtained in one-loop approximation.

Two-loop contribution $\Delta_{n}^{(2)}$ is rather cumbersome and can be found in [28]. The main and very interesting result consists in the fact that although the separated twoloop Feynman graphs of operators $F_{n}$ strongly depend on helicity parameter $\rho$, such a dependence disappears in their sum, which gives rise to critical dimensions $\Delta_{n}$. We can conclude that in two-loop approximation anomalous scaling with negative exponents (15) is not affected by the existence of non-zero helical correlations $\langle\mathbf{v} \cdot \operatorname{rot} \mathbf{v}\rangle$ in the turbulent incompressible flow. It turns out, however, that the region of stability of possible asymptotic regimes governed by fixed points of RG equations, where anomalous scaling takes place, as well as the effective diffusivity strongly depend on $\rho$. Herein below we briefly analyze the action of helicity on the effective diffusivity, which determines the exponential damping during fluctuations $\theta$.

\section{Effective diffusivity in infrared asymptotic region}

From equations for renormalization constants $Z$ (10) one immediately obtains an explicit form of $\beta$-functions and anomalous dimension $\gamma_{\nu}$ :

$$
\beta_{g} \equiv \tilde{\mathcal{D}}_{\mu} g=g\left(-2 \varepsilon-\eta+3 \gamma_{\nu}\right), \quad \beta_{u} \equiv \tilde{\mathcal{D}}_{\mu} u=u\left(-\eta+\gamma_{\nu}\right), \quad \gamma_{\nu} \equiv \tilde{\mathcal{D}}_{\mu} \ln Z_{\nu},
$$

where $\tilde{\mathcal{D}}_{\mu} \equiv \mu \partial / \partial \mu$ is logarithmic derivation at fixed bare parameters $g_{0}, u_{0}, \rho, \nu_{0}$.

From this expression and concrete calculation of $Z_{\nu}$ in two-loop approximation we can find and clasify all fixed points $g_{*}, u_{*}$ which satisfy the equations:

$$
\beta_{g}\left(g_{*}, u_{*}\right)=\beta_{u}\left(g_{*}, u_{*}\right)=0 .
$$


To investigate the infrared (IR) stability of a fixed point it is sufficient to analyze the eigenvalues of the $2 \times 2$ matrix $\Omega$ of first derivatives: $\Omega_{i j}=\partial \beta_{g_{i}} / \partial g_{j}\left(g_{i} \equiv g, u\right)$. The anomalous scaling is governed by the IR stable fixed points, i.e., those for which both eigenvalues are positive.

Classification and detailed analysis of all fixed points, determination of the region of their stability and the effect of helicity will be presented elsewhere. Here we confine ourselves to the most interesting IR stable fixed point, where both parameters $g_{*}, u_{*}$ acquire non-trivial values at $\eta=\varepsilon$ :

$$
\begin{aligned}
g_{*} & =\left(\left(g_{*}^{(1)}+\left(g_{*}^{(2)}+g_{*}^{(3)} \rho^{2}\right) \varepsilon\right) \varepsilon\right. \\
g_{*}^{(1)} & =6 \pi^{2}\left(1+u_{*}\right) u_{*} \\
g_{*}^{(2)} & =\frac{3 \pi^{2} u_{*}\left(3+u_{*}\right)}{5\left(1+u_{*}\right)^{2}} F_{1}\left(1,1 ; \frac{7}{2} ; \frac{1}{\left(1+u_{*}\right)^{2}}\right), \\
g_{*}^{(3)} & =-\frac{3 \pi^{3} u_{*}}{2}{ }_{2} F_{1}\left(\frac{1}{2}, \frac{1}{2} ; \frac{5}{2} ; \frac{1}{\left(1+u_{*}\right)^{2}}\right),
\end{aligned}
$$

where

$$
{ }_{2} F_{1}(a, b, c, z)=1+\frac{a b}{c \cdot 1} z+\frac{a(a+1) b(b+1)}{c(c+1) \cdot 1 \cdot 2} z^{2}+\cdots
$$

denotes the corresponding hypergeometric function. Actually, equation (19) represents a line of fixed points in $g-u$ plane. The competition between helical and non-helical terms appears which yields a nontrivial restriction for the fixed point values of variable $u$ to have positive fixed values for variable $g$.

Finally, let us analyze the time-behaviour of the retarded response function $G \equiv$ $\left\langle\theta \theta^{\prime}\right\rangle$ in the limit $t \rightarrow \infty$, which characterizes the response of the physical system to external regular forcing.

In frequency-wave vector representation $G(\omega, \mathbf{k})$ satisfies Dyson equation

$$
G(\omega, \mathbf{k})=\left(-\mathrm{i} \omega+\nu_{0} k^{2}-\Sigma_{\theta^{\prime} \theta}(\omega, \mathbf{k})\right)^{-1},
$$

where the self-energy operator $\Sigma_{\theta^{\prime} \theta}$ is expressed via multi-loop Feynman graphs and can be calculated perturbatively. We have found its divergent part up to two-loop approximation and calculated it exactly with a one-loop precision for some specific frequency (see below). Using (20) we find a response function in time-wave vector representation:

$$
G(t, \mathbf{k})=\int \frac{\mathrm{d} \omega}{2 \pi} \mathrm{e}^{-\mathrm{i} \omega t} G(\omega, \mathbf{k})=\int \frac{\mathrm{d} \omega}{2 \pi} \frac{\mathrm{e}^{-\mathrm{i} \omega t}}{-\mathrm{i} \omega+\nu_{0} k^{2}-\Sigma_{\theta^{\prime} \theta}(\omega, \mathbf{k})} .
$$

In the lowest approximation, $\Sigma_{\theta^{\prime} \theta}(\omega, \mathbf{k})=0$ and the integral can be easily calculated: $G_{0}(t, \mathbf{k})=\theta(t) \exp ^{-\mathrm{i} \omega_{\mathrm{r}} t}$. Here $\theta(t)$ denotes a usual step function and $\omega_{\mathrm{r}}$ is residuum in point $\mathrm{i} \omega=\mathrm{i} \omega_{\mathrm{r}}=\nu_{0} k^{2}$. According to [29] we suppose that this situation remains the same for the full response function $G$ and the leading contribution to its asymptotic behaviour as $t \rightarrow \infty$ is determined by the residuum $\omega=\omega_{\mathrm{r}}$, which corresponds to the smallest root of dispersion relation

$$
G^{-1}(\omega, \mathbf{k})=-\mathrm{i} \omega+\nu_{0} k^{2}-\Sigma_{\theta^{\prime} \theta}(\omega, \mathbf{k})=0 .
$$


Hence, the decay law $G_{0}(t, \mathbf{k}) \sim \exp ^{-\nu_{0} k^{2} t}$ is changed to

$$
G(t, \mathbf{k}) \sim \exp ^{-\mathrm{i} \omega_{\mathrm{r}} t}=\exp ^{-\nu_{\mathrm{turb}}(k) k^{2} t}, \quad t \rightarrow \infty,
$$

where turbulent diffusivity $\nu_{\text {turb }}(k) \equiv \mathrm{i} \omega_{\mathrm{r}} / k^{2}$ has been introduced. We have found this turbulent diffusivity in the scaling regime governed by the fixed point $g_{*}(19)$ :

$$
\nu_{\text {turb }}(k)=\chi D_{0}^{1 / 3} g_{*}^{-1 / 3} k^{-2 \varepsilon / 3},
$$

where $\left.\chi=1+\left(\chi_{1} \pm \chi_{2} i\right) \varepsilon\right)$ are two complex roots of dispersion relation (22). In one-loop approximation we obtain

$$
\nu_{\mathrm{turb}}(k)=\left(1+\left(\chi_{1}-\frac{g_{*}^{(2)}+g_{*}^{(3)} \rho^{2}}{3 g_{*}^{(1)}}\right) \varepsilon \pm \chi_{2} \mathrm{i} \epsilon\right)\left(\frac{D_{0}}{g_{*}^{(1)} \varepsilon}\right)^{1 / 3} k^{-2 \varepsilon / 3},
$$

where $g_{*}^{(i)}$ were defined in (19) and $\chi_{1}=4 / 3+3 \ln \left[\left(1+u_{*}\right) / 2\right], \chi_{2}=\pi / 2\left(u_{*}<\infty\right)$.

\section{Conclusions}

We have studied the advection of scalar field by turbulent flow within the framework of extended Kraichnan model and investigated the effect of helicity on anomalous scaling, stability of asymptotic regimes and the effective diffusivity. Such investigation is useful in understanding the efficiency of toy models (like Kraichnan model) in order to study the real turbulent motions by means of modern theoretical methods including renormalization group approach. Actually, we performed two-loop calculations of divergent parts of Feynman graphs, which are required to achieve multiplicative renormalization of equivalent field theoretic model. In this way we have shown that anomalous scaling, which is typical of the Kraichnan model and its numerous extensions [28,30], is not violated by inclusion of helicity to the incompressible fluid. We expect that helicity gives rise to anomalous scaling exponents in case of compressible turbulent flows. On the other hand, stability of asymptotic regimes, values of fixed $R G$ points and turbulent diffusivity strongly depend on the amount of helicity. It can be easily seen from (25) that helicity enlarges the turbulent diffusivity while high order contributions lead to the appearance of oscillations in the response function (21). We emphasize that all aforementioned effects can be observed only starting from the two-loop approximation.

\section{Acknowledgements}

M.H. is thankful to N.V.Antonov and L.Ts. Adzhemyan for discussion. The work as supported in part by VEGA grant 3211 of Slovak Academy of Sciences and by Science and Technology Assistance Agency under contract No. APVT-51-027904. 


\section{References}

1. Monin A.S., Yaglom A.M. Statistical Fluid Mechanics, vol. 2. Cambridge, MA, MIT Press, 1975.

2. McComb W.D. The Physics of Fluid Turbulence. Oxford, Clarendon, 1990.

3. Frisch U. Turbulence: The Legacy of A. N. Kolmogorov. Cambridge University Press, Cambridge, 1995.

4. Gawędzki K., Kupiainen W., Vergassola M. Program on Developed Turbulence, Erwin Schrödinger International Institute for Mathematical Physics, http://www.esi.ac.at/Programs/turbulence2002.html.

5. Zinn-Justin J. Quantum Field Theory and Critical Phenomena. Oxford, Clarendon, 1989.

6. Vasil'ev A.N. Quantum-Field Renormalization Group in the Theory of Critical Phenomena and Stochastic Dynamics. Chapman\& Hall/CRL Press, New York, 2004.

7. Bohr T., Jensen M.H., Paladin G., Vulpiani A. Dynamical Systems Approach to Turbulence. Cambridge University Press, Cambridge, 1998.

8. Bec J., Frisch U. Burgulence. - In: Les Houches 2000: New Trends in Turbulence. Eds. M. Lesieur, A. Yaglom, and F. David (Springer EDP-Sciences, 2001), p. 341-383;

E-print archives LANL nlin.CD/0012033.

9. Falkovich G., Gawędzki K., Vergassola M., Rev. Mod. Phys., 2001, 73, 913.

10. Kraichnan R.H., Phys. Rev. Lett., 1994, 72, 1016.

11. Chertkov M., Falkovich G., Kolokolov I., Lebedev V., Phys. Rev. E, 1995, 52, 4924;

Chertkov M., Falkovich G., Phys. Rev. Lett., 1996, 76, 2706.

12. Gawȩdzki K., Kupiainen A., Phys. Rev. Lett., 1995, 75, 3834;

Bernard D., Gawȩdzki K., Kupiainen A., Phys. Rev. E, 1996, 54, 2564.

13. Adzhemyan L.Ts., Antonov N.V., Vasil'ev A.N., Phys. Rev., 1998, E58, 1823;

Theor. Math. Phys., 1999, 120, 1074.

14. Adzhemyan L.Ts., Antonov N.V., Vasil'ev A.N. The Field Theoretic Renormalization Group in Fully Developed Turbulence. Gordon \& Breach, London, 1999.

15. Adzhemyan L.Ts., Antonov N.V., Barinov V.A., Kabrits Yu.S., Vasil'ev A.N., Phys. Rev. E, 2001, 63, 025303(R); 2001, 64, 019901(E);

Phys. Rev. E, 2001, 64, 056306.

16. Adzhemyan L.Ts., Antonov N.V., Hnatich M., et al., Phys. Rev. E, 2000, 63, 016309.

17. Antonov N.V., Phys. Rev. E, 1999, 60, 6691.

18. Antonov N.V., Lanotte A., Mazzino A., Phys. Rev. E, 2000, 61, 6586.

19. Adzhemyan L.Ts., Antonov N.V., Mazzino A., Muratore-Ginanneschi P., Runov A.V., Europhys. Lett., 2001, 55, 801.

20. Adzhemyan L.Ts, Vasil'ev A.N., Hnatich M., Theor. Math. Phys., 1985, 64, 777;

Theor. Math. Phys., 1987, 72, 940 (in Russian).

21. Vainstein S.I., Zel'dovich Ya.A., Ruzmaykin A.A. Turbulent dynamo in astrophysics. Nauka, Moskva, 1980 (in Russian).

22. Fedorov Yu., Katz M., Kichatinov L.L., Stehlik M., Astron. Astroph., 1992, 260, 499.

23. Katz M., Fedorov Yu., Stehlik M., Astroph. Space Sci., 1990, 166, 49.

24. Dorman L.I., Katz M., Stehlik M., BAC, 1990, 41, 312.

25. Eyink G., Phys. Rev. E, 1996, 54, 1497.

26. Bouchaud J.P., Georges A., Phys. Rep., 1990, 195, 127.

27. Honkonen J., Pis'mak Yu.M., Vasil'ev A.N., J. Phys. A, 1989, 21, 835. 
28. Adzhemyan L.Ts., Antonov N.V., Honkonen J., Phys. Rev. E, 2002, 66, 036313.

29. Adzhemyan L.V., Adzhemyan L.Ts., Vestnik Sankt Peterburgskoho Universiteta, Ser. Fiz. Khim., 2003, No. 4(28), 94 (in Russian).

30. Antonov N.V., Hnatich M., Honkonen J., Jurcisin M., Phys. Rev. E, 2003, 68, 046306.

\title{
Іграшкові моделі розвинутої турбулентності
}

\author{
М.Гнатіч, М.Юрчішін, М.Репасан
}

Інститут експериментальної фізики, Відділ теор. фізики,

Кошице, Ватсована 47, Словакія

Отримано 11 листопада 2004 р.

В рамках узагальненої моделі Крайчана нами досліджено адвекцію пасивної скалярної величини нестисливого спірального турбулентного потоку. Вважалося, що турбулентні флюктуації поля швидкості описуються гаусовою статистикою з нульовим середнім значенням та шумом із скінченими часовими кореляціями. Розрахунки виконано в наближенні двох петель у формалізмі теоретикопольової ренормалізаційної групи. Встановлено, що порушення просторового паритету (спіральність) турбулентного оточення не впливає на аномальний скейлінг, який $є$ специфічною рисою даної моделі без спіральності. Однак стабільність асимптотичних режимів, де має місце аномальний скейлінг, сильно залежить від величини спіральності. Окрім того, спіральність приводить до росту турбулентної дифузії, яка була розрахована в однопетлевому наближенні.

Ключові слова: спіральність, дифузія, аномальний скейлінг, ренормалізаційна група

PACS: $47.10 .+g, 47.27 .-i, 05.10 . C c$ 
